\title{
ON THE NUMBER OF CLOSED GEODESICS ON A RIEMANNIAN MANIFOLD
}

\author{
BY WILHELM KLINGENBERG
}

Communicated by S. S. Chern, November 19, 1963

To Kurt Reidemeister on his 70th birthday

1. Let $M$ be a compact riemannian manifold. It is a classical problem to find a lower bound for the number of closed geodesics on $M$, i.e., of curves which can be parametrized as a geodesic segment which has the same point as initial and as end point and has no corner there.

The only result known to hold for arbitrary manifolds $M$ is that there is at least one closed geodesic on $M$, cf. Fet [5], Svarc [14], Olivier [11]. To get a more precise result it will presumably always be necessary to make assumptions on the topological structure of $M$. So it is known, e.g., that a nontrivial element of the fundamental group of $M$ does determine a closed geodesic which represents this element. The case which has been studied most thoroughly so far is that $M$ is diffeomorphic to the usual $m$-sphere $S^{m}$. After preliminary results of Poincaré [12], Birkhoff [2], Lusternik-Schnirelmann [7], Morse $[9 ; 10]$ and Lyusternik [8], a rather complete answer was obtained by $\mathrm{Al}^{\prime}$ ber [1]. He assumes (as also Morse does in his work) that the diffeomorphism $\phi: M \rightarrow S^{m}$ satisfies the following condition: There is a $c>0$ such that

$$
c\|d \phi X\| \leqq\|X\|<2 c\|d \phi X\|
$$

for any tangent vector $X$ to $M$. Here, $\|Y\|$ denotes the length of the vector $Y$. Obviously, this condition may be interpreted as saying that the diffeomorphism $\phi$ shall not differ too much from the similarity with the factor $c$. Now, $\mathrm{Al}^{\prime}$ ber's result reads as follows:

Let $M$ be a compact riemannian manifold for which there exists a diffeomorphism $\phi: M \rightarrow S^{m}$ satisfying $\left(^{*}\right)$. Put $m=2^{k}+s$ with $0 \leqq s<2^{k}$. Then there exist at least $2 m-s-1$ closed geodesics on $M$ of which none is a covering of another one. All have their length in the interval $[2 \pi c, 4 \pi c[$.

The algebraic number of closed geodesics is at least $(m+1) m / 2$.

The last statement is due to Morse $[9 ; 10]$. The algebraic number of closed geodesics is obtained when counting each geodesic with a certain multiplicity. For this we refer to $\mathrm{Al}^{\prime}$ ber [1].

2. In the present paper, we announce the corresponding result for a manifold which is diffeomorphic to an arbitrary compact symmetric 
space of rank 1. To simplify the statement of the result, we restrict ourselves to the irreducible symmetric spaces and exclude the sphere which is taken care of by $\mathrm{Al}^{\prime}$ ber's result.

Let $P^{m}(\lambda), \lambda \in\{1,2,4,8\}$, be the projective space over the reals $(\lambda=1)$, the complex numbers $(\lambda=2)$, the quaternions $(\lambda=4)$ and the Cayley numbers $(\lambda=8)$, having real dimension $n=m \lambda \geqq 2 \lambda$, with $m=2$ for $\lambda=8$.

TheOREM. Let $M$ be a compact riemannian manifold for which there exists a diffeomorphism $\phi: M \rightarrow P^{m}(\lambda)$, satisfying $\left({ }^{*}\right)$. Put $m=2^{k}+s$, with $0 \leqq s<2^{k}$.

Then there exist at least $2 m-s-3+2 \lambda$ closed geodesics on $M$ of which none is a covering of another one. All have their length in the interval $[2 \pi c, 4 \pi c[$.

The algebraic number of closed geodesics on $M$ is at least $(m(m+1) / 2)(\lambda(\lambda+1) / 2)$.

Remarks. 1. If $M$ is diffeomorphic to a product of $r$ irreducible compact symmetric spaces of rank 1 , with a diffeomorphism satisfying $\left({ }^{*}\right)$, then the number of closed geodesics on $M$ is at least equal to the sum of the numbers we have obtained for the irreducible factors of the product, minus $(r-1)$.

2. Note that, for the case of the real projective space $P^{m}(1)$, we get the same number as for the sphere $S^{m}$.

3. There seems to be a possibility to extend the methods and results to cases where $M$ is diffeomorphic to a riemannian homogeneous space $H$ of a more general type than considered in the present paper. The one important property $H$ should have is that there are spheres on $H$ which would allow us to introduce great circles and circles on $H$.

3. We give a few indications of the proof of the theorem which follows, in its great lines, the proof $\mathrm{Al}^{\prime}$ ber gives for the case of the sphere. Incidentally, it seems that some points in $\mathrm{Al}^{\prime}$ ber's paper need clarification which can, however, be reached.

Denote by $P(m)=P\left(P^{m}(\lambda)\right)$ the space of piecewise differentiable closed curves on $P^{m}(\lambda)$ where all curves consisting of just one point are being identified. On $P(m)$ we have the usual metric. There are two subspaces of $P(m)$ which play an important role. The space $G(m)=G\left(P^{m}(\lambda)\right)$ of great circles on the $\lambda$-spheres in $P^{m}(\lambda)$, representing the projective lines in $P^{m}(\lambda) . G(m)$ is a manifold of real dimension $2 \lambda m-2$.

The space $C(m)=C\left(P^{m}(\lambda)\right)$ of circles on the $\lambda$-spheres in $P^{m}(\lambda)$ with all point circles being identified. $C(m)$ is a pseudomanifold of real dimension $2 \lambda m+\lambda-3$. 
We use the inclusion maps

$$
G(m) \stackrel{i_{0}}{\rightarrow} C(m) \stackrel{i}{\rightarrow} P(m)
$$

to obtain information on the singular spectral homology and cohomology groups of $P(m)$, in the sense of Čogošvili [4]. The spectral homology groups $H_{q}^{0}$ are taken with coefficients $Z_{2}$ and the discrete topology. The spectral cohomology groups $H_{0}^{q}$ are endowed with the compact open topology. $H_{q}^{0}$ is the character group of $H_{0}^{q}$ and $H_{0}^{q}$ is dense in the character group of $H_{q}^{0}$.

A main result is now: $i_{*}: H_{q}^{0}(C(m)) \rightarrow H_{q}^{0}(P(m))$ is injective and the dual homomorphism $i^{*}: H_{0}^{q}(P(m)) \rightarrow H_{0}^{q}(C(m))$ is surjective.

For the proof we have to know in detail the homology and cohomology of $G(m)$ and $C(m)$. This information is obtained with the help of intersection arguments, as introduced by Chern [3].

We recall that the length of a topological space is the maximal num. ber of cohomology classes of positive dimension which have a product $\neq 0$, plus 1, cf. Froloff-Elsholz [6] and Serre [13].

The length of $C(m)$ is found to be $2 m-s-3+2 \lambda$, with $m=2^{k}+s$, $0 \leqq s<2^{k}$. Since $i_{*}$ is injective, this number gives a lower bound for the length of $P(m)=P\left(P^{m}(\lambda)\right)$.

Using the diffeomorphism $\phi$, we now identify $M$ with $P^{m}(\lambda)$. If we talk of geodesics, of length of curves and of other properties referring to a riemannian structure on $M=P^{m}(\lambda)$, we mean the riemannian structure of $M$.

Let $z$ be a homology class $\neq 0$ in $H_{*}^{0}(P(m))$. Taking up an idea of Birkhoff [2] and Lyusternik [8], we show that $z$ determines a closed geodesic as follows: Take, among the cycles representing the homology class z, one for which a curve of maximal length has minimal length. A curve of maximal length in such a cycle then is a closed geodesic.

Different homology classes $z_{q} \in H_{q}^{0}(P(m))$ and $z_{p} \in H_{p}^{0}(P(m))$ this way might yield the same closed geodesic. However, if $z_{p}$ is subordinated to $z_{q}$, that means, if there is a cohomology class $\zeta^{q-p}$ of dimension $q-p>0$ with $z_{q} \cap \zeta^{q-p}=z_{p}$, then, according to Lyusternik 1.c., either a closed geodesic determined by $z_{q}$ has length strictly greater than the length of a closed geodesic determined by $z_{p}$, or else there exist a $(q-p)$-dimensional family of closed geodesics of the same length. It is for the proof of this fact, that the singular spectral theory of Čogošvili is being used. We have in $H_{0}(C(m))$ a sequence of $2 m-s-4+2 \lambda$ cohomology classes $\eta_{1}, \eta_{2}, \cdots$ of positive dimension which have a product $\neq 0$. Let $u$ be the fundamental class of $C(m)$. With this we form the sequence $u, u \cap \eta_{1},\left(u \cap \eta_{1}\right) \cap \eta_{2}, \cdots$ of 
$2 m-s-3+2 \lambda$ homology classes $\neq 0$. In this sequence, every homology class is subordinated to any preceding one. From the fact that the inclusion homomorphism $i_{*}: H_{*}^{0}(C(m)) \rightarrow H_{*}^{0}(P(m))$ is injective it follows that we have also in $H_{*}^{0}(P(m))$ such a sequence, with the same properties. Hence, there are at least $2 m-s-3+2 \lambda$ different closed geodesics on $M$. The fact that all these closed geodesics have their length in the interval $[2 \pi c, 4 \pi c[$ and that none is a covering of another one are simple consequences of the metric restriction $\left({ }^{*}\right)$ we did impose upon the diffeomorphism $\phi: M \rightarrow P^{m}(\lambda)$.

\section{REFERENCES}

1. S. L. Al'ber, On periodicity problems in the calculus of variations in the large, Uspehi Mat. Nauk 12 (1957), No. 4 (76), 57-124; Amer. Math. Soc. Transl. (2) 14 (1960).

2. G. D. Birkhoff, Dynamical systems with two degrees of freedom, Trans. Amer. Math. Soc. 18 (1917), 199-300.

3. S. S. Chern, On the multiplication in the characteristic ring of a sphere bundle, Ann. of Math. (2) 49 (1948), 362-372.

4. G. S. Čogošvili, On spectrally singular homology groups, Soobšč. Akad. Nauk Gruzin. SSR 14 (1953), 383-388. (Russian)

5. A. I. Fet, Variational problems on closed manifolds, Mat. Sb. (N.S.) 30 (72) (1952), 271-361 (Russian); Amer. Math. Soc. Transl. no. 90 (1953).

6. S. V. Froloff and L. E. Elsholz, Limite inférieure pour le nombre des valeurs critiques d'une fonction, donnée sur variêté, Mat. Sb. (N.S.) 42 (1935), 637-643.

7. L. Lusternik and L. Schnirelmann, Existence de trois lignes gêodésiques fermées sur la surface de genre O, C. R. Acad. Sci. Paris 188 (1929), 269-271.

8. L. A. Lyusternik, The topology of function spaces and the calculus of variations in the large, Trudy Mat. Inst. Steklov 19 (1947). (Russian)

9. M. Morse, Closed extremals, Proc. Nat. Acad. Sci. U.S.A. 15 (1929), 856-859.

10. - The calculus of variations in the large, Amer. Math. Soc. Colloq. Publ. Vol. 18, Amer. Math. Soc., Providence, R. I., 1934.

11. R. Olivier, Die Existenz geschlossener Geodätischer auf kompakten Mannigfaltigkeiten, Comment. Math. Helv. 35 (1961), 146-152.

12. H. Poincaré, Sur les lignes géodésiques des surfaces convexes, Trans. Amer. Math. Soc. 6 (1905), 237-274.

13. J.-P. Serre, Homologie singulière des espaces fibrés, applications, Ann. of Math. (2) 54 (1951), 425-505.

14. A. S. Švarc, The homology of some spaces of mappings, Memoirs of the 3rd Soviet Mathematical Congress, Vol. II, Moscow, 1956, p. 137, Izdat. Akad. Nauk SSSR, Moscow, 1956. (Russian)

\section{Mathematisches Institut, Universität Mainz, Germany and Instituto de Física e Matematica, Recife, Brazil}

